\title{
Multi-Scale Modeling of the Gas-Liquid Interface Based on Mathematical and Thermodynamic Approaches
}

\author{
Yukihiro Yonemoto $^{1, *}$ and Tomoaki Kunugi ${ }^{2}$ \\ ${ }^{I}$ Department of Applied Electronics, Faculty of Industrial Science and Technology, Tokyo University of Science, Yama- \\ saki 2641, Noda, Chiba, 278-8510, Japan \\ ${ }^{2}$ Department of Nuclear Engineering, Kyoto University, Yoshida, Sakyo, Kyoto, 606-8501, Japan
}

\begin{abstract}
A gas-liquid interface involves complex physics along with unknown phenomena related to thermodynamics, electromagnetics, hydrodynamics, and heat and mass transfer. Each phenomenon has various characteristic time and space scales, which makes detailed understanding of the interfacial phenomena very complex. Therefore, modeling the gasliquid interface is a key issue for numerical research on multiphase flow. Currently, the continuum surface force (CSF) model is popular in modeling the gas-liquid interface in multiphase flow. However, the CSF model cannot treat the various chemical and physical phenomena at the gas-liquid interface because it is derived based only on mechanical energy balance and it assumes that the interface has no thickness. From certain experimental observations, bubble coalescence/repulsion was found to be related to a contamination at the interface.

The present study developed a new gas-liquid interfacial model based on thermodynamics via a mathematical approach, assuming that the interface has a finite thickness like a thin fluid membrane. In particular, free energy, including an electrostatic potential due to the contamination at the interface, is derived based on a lattice gas model. Free energy is incorporated into the conventional Navier-Stokes equation as new terms using Chapman-Enskog expansion based on the multiscale concept. Using the Navier-Stokes equation with the free energy terms, we derived a new governing equation of fluid motion that characterizes mesoscopic scale phenomena. Finally, the new governing equation was qualitatively evaluated by simulating an interaction between two microbubbles in two dimensions while also accounting for electrostatic force.
\end{abstract}

Keywords: Multiphase flow, multi-scale modeling, bubble coalescence, contamination.

\section{INTRODUCTION}

A gas-liquid interface is geometrically characterized by a curvature at a macroscopic scale in which vortical fluid motion is dominant. This indicates that the interface is considered as a discontinuous surface without interfacial thickness. Hence, physical properties such as density, viscosity, and temperature have discontinuous values at the interface. On the other hand, at a microscopic scale such as that of molecular motion, the physical properties continuously vary from the gas phase to the liquid phase. Currently, there are no adequate experimental measurement techniques to clarify the complexity of the interface. Therefore, a numerical approach is a useful tool for studying gas-liquid interfacial phenomena. However, modeling the interface while covering the wide range of scales from molecular motion to vortical fluid motion is very difficult. This has been the key issue of numerical research on multiphase flow for many years.

Numerical analysis of multiphase flow is generally performed by combining an interfacial model with the NavierStokes (NS) equation. The continuum surface force (CSF) model [1] is a popular interfacial model for the gas-liquid

\footnotetext{
*Address correspondence to this Department of Applied Electronics, Faculty of Industrial Science and Technology, Tokyo University of Science, Yamasaki 2641, Noda, Chiba, 278-8510, Japan; Tel: +81-4-7124-1501; Fax: +81-4-7122-1499; E-mail: yonemoto@ @e.noda.tus.ac.jp
}

interface. Surface tension is incorporated into the NS equation as a body force across the interface. The volume of fluid (VOF) method [2] is used with the CSF model as an interface tracking method. In the VOF method, the interface is recognized by the fractional volume of fluid denoted by a fraction of density. Matsumoto et al. [3] performed a numerical simulation of bubbly flow based on the VOF method with the CSF model. Their study reported that bubble coalescence occurs easily. In experimental observations, it is known that the behavior of bubble coalescence is not as simple as predicted in numerical simulations. This non-realistic bubble coalescence may arise from the feature of the CSF model; the CSF model cannot consider the various physical and chemical processes at the gas-liquid interface because it is derived based only on mechanical energy balance and assumes that the gas-liquid interface has no thickness (i.e., a mathematical interface).

Tryggvason et al. [4] developed the front tracking method based on both Eulerian and Lagrangian concepts, which uses the fixed structured numerical grids in the flow field. Their approach treats the gas-liquid interface using an unstructured grid. In this model, the interface is represented by line segments in two dimensions or polygonal elements in three dimensions. Surface tension is evaluated using differential geometry; a tangential vector is defined on the interface. The front tracking method is also based on the mathe- 
matical interface and cannot consider bubble coalescence or breakup naturally. Artificial criteria, in particular, are needed to account for the process of coalescence; however, much success has been achieved in simulating bubbly flow and bubble behavior [5].

Although there are many models or methods for simulating interfacial behavior [6-8], these approaches face difficulty in considering the physical and chemical processes at the interface because they are also based on the conventional mechanical approach.

Recently, the phase field theory [9] and the van der Waals theory [10], which assume the interface to be a diffuse interface with finite thickness, have been applied to numerical research on multiphase flow. The surface force is evaluated using the free energy defined at the interface and depends on both concentration (or density) and its gradient. A numerical method based on the phase field theory (called the "phase field method") focuses on an analysis of mesoscopic phenomena and was originally used in studies on diffusivity of grain growth, or phase separation, in binary alloys [1114]. A chemical potential is associated with the free energy and a diffusion flux is assumed to be proportional to the chemical potential gradient $[15,16]$. The time evolution of the concentration associated with the diffusion flux is implemented using the Cahn-Hilliard equation. Jacqmin [17, 18] applied the phase field method to two-phase flow analysis. Various interfacial phenomena were simulated, such as interfacial interactions (coalescences and breakups), contact line movement, and interfacial instability. The phase field method based on the phase field theory is well constructed thermodynamically and holds potential for investigating physical and chemical phenomena, and heat and mass transfer, at a gas-liquid interface. However, the phase field method is the fundamental numerical tool to investigate phenomena such as diffusivity of metal alloys. Therefore, a crucial issue arises in the application of the phase field method to multiphase flow analysis. The dynamic motion of the interface in a fluid flow does not result from a diffusion process characterized by mobility.

A contamination involving electric charges at the interface is significant to the interfacial interaction. Craig [19] and Henry and Craig [20] studied the effects of a specific ion on bubble interaction. They reported that bubble coalescence is affected by the adsorbed ions (combination of ions and electrolyte concentration) at the gas-liquid interface. Similarly, other literature reports the importance of an electrolyte for bubble coalescence [21-24]. To elucidate the detailed mechanism of bubble coalescence/repulsion, we need a new gas-liquid interfacial model that can investigate the physical, chemical, and various other phenomena at the interface characterized by different time and space scales.

In the present study, the thermodynamic and mathematical interfacial model was developed based on the phase field theory [9] and a multi-scale concept. This model assumes that the interface has a finite thickness similar to a fluid membrane. Essentially, the free energy including the electric charge due to a contamination is derived at the microscopic scale. The free energy is incorporated into the NS equation as new terms, using Chapman-Enskog expansion [25]. A new governing equation of fluid motion is then derived. Finally, we qualitatively evaluate the new governing equation and its potential in the future realization of a multi-scale simulation by simulating an interaction between two microbubbles in two dimensions while also accounting for the electrostatic force.

\section{FREE ENERGY WITH ELECTROSTATIC POTEN- TIAL}

Free energy is considered at the microscopic scale, and the specific equation of free energy is derived by considering a Hamiltonian [26]. In the present derivation, an electrostatic potential due to a contamination at the interface is considered.

\subsection{Hamiltonian}

Although the Ising model explains a phase transition of a magnetic material in a magnetic field, this model is also applicable to a gas-liquid phase transition; it is called the "lattice gas model."

Consider a three-dimensional cubic lattice in a binary system in which the lattice is $l[\mathrm{~m}]$ in width. A variable $\sigma_{i}$ (or $\sigma_{j}$ ) [-] is defined at the lattice point $\{i\}$ (or $\{j\}$ ) and takes the value of 0 or 1 ; the value represents the existence of molecule A or B in the lattice gas model, respectively. Fig. (1) shows a schematic of a two-dimensional lattice for the sake of simplicity. The following two assumptions are considered in the lattice gas model:

(a)

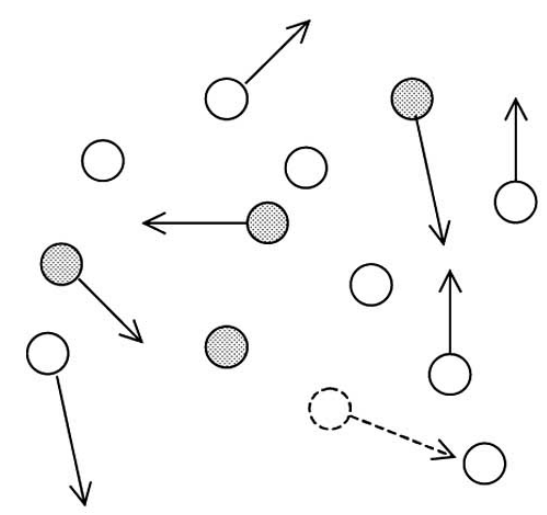

(b)

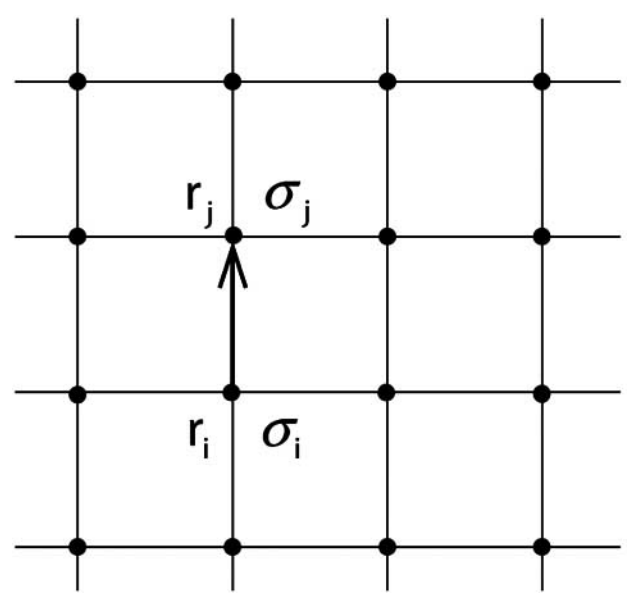

Fig. (1). Schematic diagram of lattice gas model. $\sigma_{i}=0 \rightarrow$ Molecule $A, \sigma_{i}=1 \rightarrow$ Molecule $B, l=\left|\boldsymbol{r}_{j}-\boldsymbol{r}_{i}\right|$. (a) Molecular motion in physical space. (b) Mathematical model of molecular motion. 
1. Only one molecule exists at a lattice point $\{i\}$ (or $\{j\}$ ).

2. Only two-body interaction is considered between molecules A and B. tions:

In addition, the molecule satisfies the following condi-

3. The size and shape of both molecules A and B are the same.

4. The intermolecular force is only a short-range force (i.e., van der Waals attraction).

The liquid solution satisfying conditions 3 and 4 is called the "regular solution." This study considers the electrostatic potential due to a contamination at the interface. However, the adsorption process of the contamination is ignored for the sake of simplicity.

The gas-liquid interface may be electrically charged by the contamination, such as specific adsorption of the solvent molecules. In the present study, the electrostatic potential created by the contamination at the interface is modeled as an electric double layer that may be formed near the interface. Here, we assume that the electric effect on the solvent molecules in the electric double layer can be considered as similar to the effect of a spatially distributed electric potential on a point charge. Based on the above assumptions, a Hamiltonian $H[\mathrm{~J}]$ can be defined at the lattice point $\{i\}$ with the electrostatic potential $E[\mathrm{~J}]$ as follows:

$$
\begin{aligned}
& H=-\frac{1}{2} \sum_{i j} Y_{i j}+E, \\
& E=-\frac{1}{2} \sum_{i}^{N} z_{i} e V_{e}\left(\boldsymbol{r}_{i}\right) \sigma_{i}, \\
& Y_{i j}=W_{i j}^{A A}\left(1-\sigma_{i}\right)\left(1-\sigma_{j}\right)+W_{i j}^{B B} \sigma_{i} \sigma_{j}, \\
& +W_{i j}^{A B}\left\{\sigma_{i}\left(1-\sigma_{j}\right)+\sigma_{j}\left(1-\sigma_{i}\right)\right\}, \\
& \sum_{i j}=\sum_{i}^{N} \sum_{j}^{N} .
\end{aligned}
$$

Here, $N$ denotes the total number of lattice points; $z_{i}[-], e$ [C], and $V_{e}[\mathrm{~V}]$ are valence, elementary charge, and electric potential, respectively. Intermolecular potential is represented by $W_{i j}[\mathrm{~J}]$ (positive value). The superscript of $W_{i j}$ represents a combination of interaction between molecules; $W_{i j}^{A B}$ is an intermolecular potential between molecules $\mathrm{A}$ and $\mathrm{B}$. Equation (3) reduces to the following equation under assumptions 1, 2, 3, and 4:

$Y_{i j}=W_{i j}^{A A}-\left(W_{i j}^{A A}+W_{i j}^{B B}-2 W_{i j}^{A B}\right) \sigma_{i}\left(1-\sigma_{j}\right)$.

Now let $U_{i j}$ be a net interaction potential; thus

$U_{i j}=W_{i j}^{A A}+W_{i j}^{B B}-2 W_{i j}^{A B}$.

Considering Equations (5) and (6), Equation (1) reduces to the following equation:

$H=\frac{1}{2} \sum_{i j} U_{i j} \sigma_{i}\left(1-\sigma_{j}\right)-\frac{1}{2} \sum_{i}^{N} z_{i} e V_{e}\left(\boldsymbol{r}_{i}\right) \sigma_{i}$.
This is the Hamiltonian that includes the electrostatic potential due to the contamination.

\subsection{Selection of Free Energy}

Consider a system described by the exact Hamiltonian (Equation (7)); henceforth, such a system is called an "exact system." It is impossible to get an exact partition function with respect to the exact Hamiltonian because of a multibody system. Instead we employ an approximation technique using the variational theorem of Bogoliubov and mean field theory as a general method to solve the problem in the multibody system [27]. To obtain the free energy equation in the exact system, the approximation technique needs a certain criterion; the Bogoliubov inequality criterion is stated as follows:

$$
F_{Z} \leq F_{Z_{0}}+\left\langle H_{Z}-H_{Z_{0}}\right\rangle_{0}
$$

where, $F_{Z}$ and $H_{Z}$ are the Helmholtz free energy and the Hamiltonian, respectively, in the exact system. $F_{Z_{0}}$ and $H_{Z_{0}}$ are the Helmholtz free energy and the Hamiltonian, respectively, in a model system, where the free energy and partition function are known. $H_{Z}-H_{Z_{0}}$ in the second term on the right hand side of Equation (8) is averaged by the distribution function in the model system. Equation (8) states that the free energy $F_{Z}$ can be approximated by the solutions of an effective Hamiltonian $H_{Z_{0}}$. Here, the Helmholtz free energy $F_{Z_{0}}$ and the partition function $Z_{0}$ are as follows:

$$
\begin{aligned}
& F_{Z_{0}}=-k_{B} T \ln Z_{0}, \\
& Z_{0}=\int_{\Gamma} \exp \left(-\frac{H_{Z_{0}}}{k_{B} T}\right) \mathrm{d} \bar{\Gamma}, \\
& \mathrm{d} \bar{\Gamma}=\frac{\mathrm{d} \boldsymbol{p} \mathrm{d} \boldsymbol{q}}{(2 \pi \hbar)^{3}},
\end{aligned}
$$

where $\mathrm{d} \bar{\Gamma}$ is an infinitesimal volume in a phase space $\bar{\Gamma}$, which consists of a space coordinate $\boldsymbol{p}$ and a momentum $\boldsymbol{q}$. In Equation (11), the value $(2 \pi \hbar)^{3}$ is the coefficient used to normalize the distribution function and represents a small volume in $\bar{\Gamma}$ space. This normalization is based on the consideration of the quantum uncertainty $\mathrm{d} p_{i} \mathrm{~d} q_{j} \cong 2 \pi \hbar(i, j=1$, $2,3)$; $\hbar$ is $h / 2 \pi$, where $h[\mathrm{~J} \cdot \mathrm{s}]$ is the Plank's constant.

The free energy in the exact system is obtained by minimizing the right hand side of Equation (8). The Helmholtz free energy $F_{Z_{0}}$ is determined using Equation (7) based on the mean field theory. This means that the bilinear form $\sigma_{i} \sigma_{j}$ in Equation (7) is replaced by the primary form $\beta_{i} \sigma_{i}$ in which the coefficient $\beta_{i}$ is the parameter used to minimize the right hand side of Equation (8). Thus, the Hamiltonian $H_{Z_{0}}$ in the model system becomes

$$
H_{Z_{0}}=\sum_{i}^{N} k_{B} T \beta_{i} \sigma_{i}-\frac{1}{2} \sum_{i}^{N} z_{i} e V_{e}\left(\boldsymbol{r}_{i}\right) \sigma_{i}
$$


where $k_{B}\left[\mathrm{~m}^{2} \mathrm{~kg} / \mathrm{s}^{2} \mathrm{~K}\right]$ and $T[\mathrm{~K}]$ are the Boltzmann constant and temperature, respectively. By considering Equation (12) and $\sigma_{i}=0$ or 1 , Equation (10) is transformed into the following equation:

$Z_{0}=\prod_{i}^{N} \sum_{\sigma_{i}=1,0} \exp \left(-\bar{\beta}_{i} \sigma_{i}\right)$

$\bar{\beta}_{i}=\beta_{i}-\frac{z_{i} e V_{e}}{2 k_{B} T}$.

Substitution of Equation (13) into Equation (9) yields

$$
F_{Z_{0}}=-\sum_{i} k_{B} \operatorname{Tn}\left(\exp \left(-\bar{\beta}_{i}\right)+1\right) \text {. }
$$

Criterion (8) holds between the exact and the model systems. Thus, the substitution of Equations (7), (9), and (12) into Equation (8) yields

$$
F_{Z} \leq-k_{B} T \ln Z_{0}+\frac{1}{2} \sum_{i j} U_{i j}\left\langle\sigma_{i}\left(1-\sigma_{j}\right)\right\rangle_{0}-\sum_{i}^{N} k_{B} T \beta_{i}\left\langle\sigma_{i}\right\rangle_{0} .
$$

The second term on the right hand side of this equation is the linearized term with respect to $\sigma_{i}$ in the model system. Therefore, the interaction $\left\langle\sigma_{i} \sigma_{j}\right\rangle_{0}$, which is the value of $\sigma_{i} \sigma_{j}$ averaged by the distribution function $Z_{0}$ in the model system, can be decomposed to $\left\langle\sigma_{i}\right\rangle_{0}\left\langle\sigma_{j}\right\rangle_{0}$. Eventually, Equation (16) reduces to the following equation:

$$
F_{Z} \leq-k_{B} T \ln Z_{0}+\frac{1}{2} \sum_{i j} U_{i j}\left\langle\sigma_{i}\right\rangle_{0}\left(1-\left\langle\sigma_{j}\right\rangle_{0}\right)-\sum_{i}^{N} k_{B} T \beta_{i}\left\langle\sigma_{i}\right\rangle_{0},(17)
$$

where the parameter $\left\langle\sigma_{i}\right\rangle_{0}$ takes continuous values from 0 to 1. By selecting the parameter $\beta_{i}$ to minimize the right hand side of Equation (17), the best solution in the exact system can be obtained. The calculation of the functional derivative of Equation (17) keeping $\left\langle\sigma_{i}\right\rangle_{0}$ constant and $\delta F_{Z} / \delta \beta_{i}=0$ yields

$\left\langle\sigma_{i}\right\rangle_{0}=\frac{\exp \left(-\bar{\beta}_{i}\right)}{\exp \left(-\bar{\beta}_{i}\right)+1}$.

As $\left\langle\sigma_{i}\right\rangle_{0}=\phi_{i}$, the specific formula of $\beta_{i}$ is obtained from Equation (18) by considering Equation (14). Then, the substitution of $\beta_{i}$ into Equation (17) yields

$$
\begin{aligned}
& F_{Z}=\sum_{i}^{N} k_{B} T\left\{\left(1-\phi_{i}\right) \ln \left(1-\phi_{i}\right)+\phi_{i} \ln \phi_{i}\right\}- \\
& \sum_{i}^{N} \frac{1}{2} z_{i} e V_{e} \phi_{i}+\frac{1}{2} \sum_{i j} U_{i j} \phi_{i}\left(1-\phi_{j}\right)
\end{aligned}
$$

This is the free energy equation in the exact system and the discrete equation of free energy using the local continuous function $\phi_{i}$. Using the thermodynamic procedures discussed above, the molecular state represented by 0 or 1 shown in
Fig. (2a) becomes the local continuous state shown in Fig. (2b). However, the local continuous state is still at the microscopic spatial scale. Thus, a continuum limit is applied mathematically to Equation (19) for obtaining the free energy equation of the continuous spatial scale. Consequently, the discrete state shown in Fig. (2b), where the lattice width $l$ is defined as a finite length, becomes the continuous state shown in Fig. (2c), where the finite length of $l$ approaches 0 infinitely.

(a)

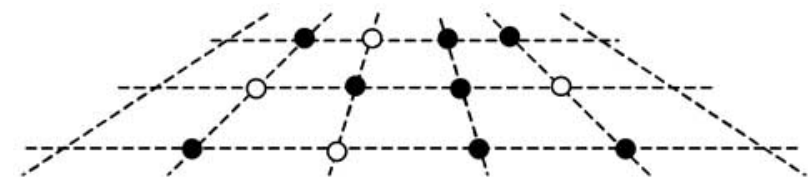

(b)

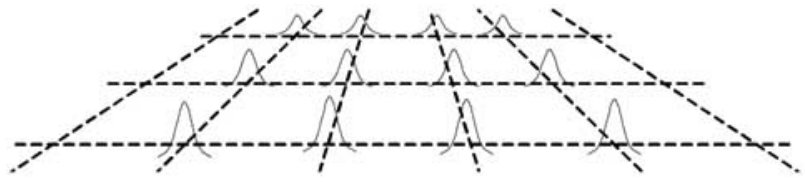

(c)

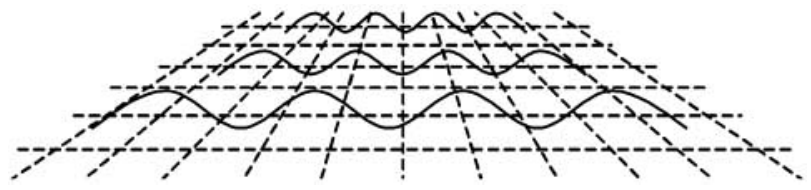

Fig. (2). Schematic diagram of space scale from discrete points to continuum: (a) shows lattice points (distribution of $\sigma_{i}$ ) in which filled circles denote molecule A and empty circles denote molecule B; (b) shows local continuum (distribution of $\phi_{i}$ ); and (c) shows continuum limit (distribution of $\phi$ ).

The third term on the right hand side of Equation (19) is the interaction between the lattices $i$ and $j$. In particular, the third term is transformed as follows:

$\frac{1}{2} \sum_{i j} U_{i j} \phi_{i}\left(1-\phi_{j}\right)=\frac{1}{4} \sum_{i j} U_{i j}\left\{\left(\phi_{i}-\phi_{j}\right)^{2}-\phi_{i}^{2}-\phi_{j}^{2}+2 \phi_{i}\right\}$.

If the interaction between the nearest neighboring molecules is just the short-range interaction, assuming that the distance of the closest approach is $l$, the first term on the right hand side of Equation (20) is transformed by the continuum limit $(l \rightarrow 0)$ as follows:

$\left(\phi_{i}-\phi_{j}\right) \boldsymbol{n}=\left(l \frac{\phi_{i}-\phi_{j}}{l}\right) \boldsymbol{n} \underset{l \rightarrow 0}{\rightarrow} l \nabla \phi$,

where $\phi_{i} \rightarrow \phi, \phi_{j} \rightarrow \phi$, and $\boldsymbol{n}$ is the unit vector between lattice $\{i\}$ and $\{j\}$. In addition, by the continuum limit $(N \rightarrow \infty, l \rightarrow 0)$ we obtain

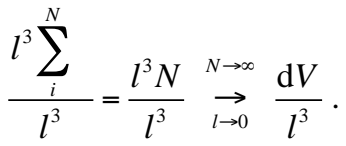

Consequently, by considering Equations (20), (21), and (22), Equation (19) becomes 


$$
F=\int_{V}\left[f_{0}(\phi)+\frac{S_{0}}{2}(\nabla \phi)^{2}\right] \mathrm{d} V .
$$

Here,

$$
\begin{aligned}
& f_{0}(\phi)=\frac{1}{l^{3}}\left[k_{B} T\{\phi \ln \phi+(1-\phi) \ln (1-\phi)\}+\frac{U_{\text {sum }}}{2} \phi(1-\phi)\right], \\
& -\frac{1}{2 l^{3}} z_{i} e V_{e} \phi \\
& S_{0}=\frac{U_{\text {sum }}}{2 l^{3}} \\
& U_{\text {sum }}=\sum_{j}^{N} U_{i j} .
\end{aligned}
$$

This is the free energy equation of the continuous scale in an infinitesimal volume, $\mathrm{d} V\left[\mathrm{~m}^{3}\right]$. In a narrow sense, this equation is called the "free energy functional." The first and second terms on the right hand side of Equation (23) are the free energies in a homogeneous and an inhomogeneous system, respectively. Here, the fourth-order Taylor expansion of Equation (23) around $\phi=1 / 2(\psi=\phi-1 / 2)$ gives

$$
F=\int_{V}\left[f_{0}(\psi)+\frac{d}{2}(\nabla \psi)^{2}\right] \mathrm{d} V .
$$

Here,

$$
\begin{aligned}
& f_{0}(\psi)=-\frac{a}{2} \psi^{2}+\frac{b}{4} \psi^{4}-c z_{i} e V_{e} \psi, \\
& a=\frac{U_{\text {sum }}-4 k_{B} T}{l^{3}},
\end{aligned}
$$

$b=\frac{16 k_{B} T}{3 l^{3}}$,

$c=\frac{1}{2 l^{3}}$,

$d=\frac{U_{\text {sum }}}{2 l^{3}}$.

This expansion of free energy is called Ginzburg-Landau expansion and is generally applied to the critical state $(\phi=1 / 2)$. However, we assume that the same expansion can be applied to a state of $\phi=1 / 2$ in the interface, even if the system is at room temperature. $\psi$ is an order parameter that characterizes the exact system, and is mainly characterized by the concentration or density.

\section{INTERPRETATION OF FREE ENERGY IN THE NAVIER-STOKES EQUATION}

An interpretation and a treatment of the free energy equation in the NS equation are discussed here. In general, surface tension is evaluated by the Young-Laplace equation, which consists of the surface tension coefficient and the curvature characterizing the macroscopic shape of the interface (Fig. (3a)). However, at the microscopic scale, molecular motion is continuous in the interface. The present model assumes that the interface has a finite thickness similar to a fluid membrane. This assumption suggests a mesoscopic interface, as shown in Fig. (3b). For a finite-thickness interface, it is difficult to evaluate surface tension because the curvature cannot be determined geometrically. Therefore, surface tension should be evaluated not from the geometrical curvature but from the energy at such a mesoscopic scale $[28,29]$. Furthermore, we cannot simply incorporate free energy into the NS equation as an external force because many physical and chemical processes characterized by different time and space scales occur at the interface. These cross-scale interactions may arise among interfacial phenomena. Thus, the multi-scale concept must be considered when free energy is incorporated into the NS equation. Bearing this in mind, Chapman-Enskog expansion is applied to the NS equation. Here, the original NS equation is expressed as follows:

(a)

(b)
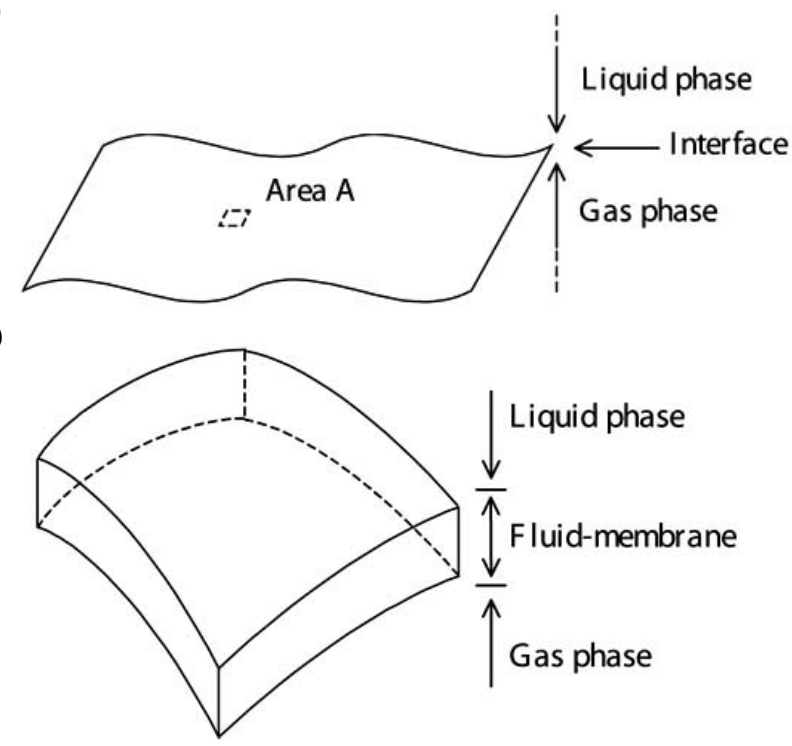

Fig. (3). Concept of gas-liquid interface. (a) Macroscopic image. (b) Mesoscopic image of Area A.

$\rho \frac{\mathrm{D} \boldsymbol{u}}{\mathrm{D} t}=-\nabla \cdot \boldsymbol{T}+\rho \boldsymbol{g}$,

where $\rho\left[\mathrm{kg} / \mathrm{m}^{3}\right], \boldsymbol{u}[\mathrm{m} / \mathrm{s}], \boldsymbol{T}\left[\mathrm{N} / \mathrm{m}^{2}\right], \boldsymbol{g}\left[\mathrm{m} / \mathrm{s}^{2}\right]$, and $t[\mathrm{~s}]$ represent fluid density, velocity, stress tensor, acceleration due to gravity, and time, respectively. The operators of $\mathrm{D} / \mathrm{D} t$ and $\boldsymbol{\nabla}$ have to include various time and space scales (this is a multi-scale concept). Therefore, to discriminate their scales, Chapman-Enskog expansion is applied to $\mathrm{D} / \mathrm{D} t$ and $\boldsymbol{\nabla}$. The operators $\mathrm{D} / \mathrm{D} t$ and $\boldsymbol{\nabla}$ are transformed into the following expressions using a small parameter $\varepsilon$ :

$$
\begin{aligned}
& \boldsymbol{\nabla}=\boldsymbol{\nabla}^{(0)}+\varepsilon \boldsymbol{\nabla}^{(1)}+\varepsilon^{2} \boldsymbol{\nabla}^{(2)}+\cdots+\varepsilon^{k} \boldsymbol{\nabla}^{(k)}+\cdots, \\
& \frac{\mathrm{D}}{\mathrm{D} t}=\frac{\mathrm{D}}{\mathrm{D} t^{(0)}}+\varepsilon \frac{\mathrm{D}}{\mathrm{D} t^{(1)}}+\varepsilon^{2} \frac{\mathrm{D}}{\mathrm{D} t^{(2)}}+\cdots+\varepsilon^{k} \frac{\mathrm{D}}{\mathrm{D} t^{(k)}}+\cdots,
\end{aligned}
$$

where superscript $(k)(k=0,1,2 \ldots)$ represents the scale of the phenomena. The scale decreases as the value of $(k)$ increases; e.g., the superscript (0) denotes the macroscopic scale. The parameter $\varepsilon$ is defined as follows: 
$\varepsilon=\frac{\delta}{L}$.

Here, $\delta[\mathrm{m}]$ and $L[\mathrm{~m}]$ represent the characteristic length of the interface and that of the vortical fluid flow, respectively. The substitution of Equations (34) and (35) into Equation (33) and consideration of $\varepsilon$ up to the first order yields

$\rho \frac{\mathrm{D} \boldsymbol{u}}{\mathrm{D} t^{(0)}}+\varepsilon \rho \frac{\mathrm{D} \boldsymbol{u}^{\prime}}{\mathrm{D} t^{(1)}}=-\nabla^{(0)} \cdot \boldsymbol{T}-\varepsilon \boldsymbol{\nabla}^{(1)} \cdot \boldsymbol{T}^{\prime}+\rho \boldsymbol{g}$,

where the fluid velocity and the stress tensor in the macroscopic scale are represented by $\boldsymbol{u}$ and $\boldsymbol{T}$, respectively. On the other hand, the physical values in the mesoscopic scale are represented by $\boldsymbol{u}^{\prime}$ and $\boldsymbol{T}^{\prime}$, respectively. The stress tensor $\boldsymbol{T}$ is defined as follows:

$\boldsymbol{T}=P \boldsymbol{I}-\boldsymbol{\tau}$.

In this equation, the shear stress is $\tau\left[\mathrm{N} / \mathrm{m}^{2}\right]$. The pressure $P\left[\mathrm{~N} / \mathrm{m}^{2}\right]$ is the mechanical pressure, hereafter represented by $P_{\text {mech }}$. The velocity $\boldsymbol{u}^{\prime}$ and the stress tensor $\boldsymbol{T}^{\prime}$ in Equation (37) correspond to the physical values at the interface. In the present study, the stress tensor is represented by the Maxwell relation. Therefore, thermodynamic pressure $P_{\text {therm }}$ is defined as follows:

$\boldsymbol{P}_{\text {therm }}=-\left(\frac{\partial F}{\partial V}\right)_{T} \boldsymbol{I}$.

In Equation (39), $F$ is the Helmholtz free energy. Assuming that Equation (39) corresponds to $\boldsymbol{T}^{\prime}$, Equation (39) becomes the following equation using Equation (27):

$\boldsymbol{T}^{\prime}=\boldsymbol{P}_{\text {therm }}=-\left[f_{0}(\psi)+\frac{d}{2}\left(\nabla^{(1)} \psi\right)^{2}\right] \boldsymbol{I}$

Then, Equation (40) is substituted into Equation (37). After the simple tensor analysis of the term $-\varepsilon \boldsymbol{\nabla}^{(1)} \cdot \boldsymbol{T}^{\prime}$, the following equation is obtained:

$$
\begin{aligned}
& -\varepsilon \boldsymbol{\nabla}^{(1)} \cdot \boldsymbol{T}^{\prime}=\varepsilon \boldsymbol{\nabla}^{(1)} \cdot\left(f_{0}(\psi) \boldsymbol{I}\right)+\varepsilon d \boldsymbol{\nabla}^{(1)} \cdot\left(\boldsymbol{\nabla}^{(1)} \psi \otimes \boldsymbol{\nabla}^{(1)} \psi\right)-\varepsilon d \boldsymbol{\nabla}^{(1)} \psi \\
& \left(\boldsymbol{\nabla}^{(1)} \cdot \boldsymbol{\nabla}^{(1)} \psi\right)=\varepsilon \boldsymbol{\nabla}^{(1)} \cdot(\underbrace{P_{\mathrm{int}} \boldsymbol{I}}_{(a)}+\underbrace{\boldsymbol{\tau}_{\mathrm{int}}}_{(b)}) \underbrace{-\varepsilon d \boldsymbol{\nabla}^{(1)} \psi\left(\boldsymbol{\nabla}^{(1)} \cdot \boldsymbol{\nabla}^{(1)} \psi\right)}_{(c)} .
\end{aligned}
$$

In Equation (41), term (a) denotes the free energy in a homogeneous system. This term is concerned with changes of state and is important when a phase change is considered. Term (b), $\boldsymbol{\tau}_{\text {int }}=d\left(\boldsymbol{\nabla}^{(1)} \psi \otimes \boldsymbol{\nabla}^{(1)} \psi\right)$, is taken as the shear stress in the interface. The additional term (c) represents surface tension. Here, we assume that the non-diagonal components of $\boldsymbol{\tau}_{\text {int }}$ are negligible in the interface because the thickness of the interface is significantly small compared to a minimum scale of an eddy such as the Kolmogorov scale. In addition, the diagonal components of $\boldsymbol{\tau}_{\text {int }}$ are negligible because a mean pressure balances out at the interface. Eventually, after the substitution of Equation (41) into Equation (37), a new governing equation of fluid motion considering the multiscale concept is obtained as follows:

$$
\begin{aligned}
& \rho \frac{\mathrm{D} \boldsymbol{u}}{\mathrm{D} t^{(0)}}+\varepsilon \rho \frac{\mathrm{D} \boldsymbol{u}^{\prime}}{\mathrm{D} t^{(1)}}=-\boldsymbol{\nabla}^{(0)} \cdot \boldsymbol{T}+\varepsilon \boldsymbol{\nabla}^{(1)} \cdot\left(f_{0}(\psi) \boldsymbol{I}\right) \\
& -\varepsilon d \boldsymbol{\nabla}^{(1)} \psi\left(\boldsymbol{\nabla}^{(1)} \cdot \boldsymbol{\nabla}^{(1)} \psi\right)+\rho \boldsymbol{g}
\end{aligned} .
$$

We call this equation the multi-scale multiphase flow equation. The surface tension formalism involves the combination of coefficient $d$ and the interfacial gradient. The surface tension formalism in the present model resembles that of the CSF model. However, the interpretations of the surface tensions of the CSF model and the present model are a little different. The difference is explained by a simple consideration of term (c) in Equation (41). The surface tension of the CSF model is estimated by the Young-Laplace equation, in which the interface has no thickness (i.e., a mathematical interface). In the CSF model, the coefficient corresponds to the surface tension coefficient measured experimentally. On the other hand, in the present model, term (c) is derived based on the thermodynamic approach in which the interface has a finite thickness. The microscopic coefficient $d$ in term (c) is derived at a microscopic scale and is related to the intermolecular interaction. Here, we assume that the intermolecular interaction can be estimated approximately by the following equation [30]:

$$
U_{i j}=-\frac{A}{6 h_{i j}}\left(\frac{r_{i} r_{j}}{r_{i}+r_{j}}\right) \text {. }
$$

Here, $A, r_{i}$ or $r_{j}$, and $h_{i j}$ are the Hamaker constant, radius of molecules, and distance between two molecules, respectively. The order of magnitude of coefficient $d$ is very small (about $10^{-11}[\mathrm{~N}]$ ). In thermodynamics [9, 10], the surface tension coefficient is obtained by integrating the free energy through a flat interface with a finite thickness. The gradient of free energy at the interface is very large. Therefore, term (c) can act as surface tension by considering both the gradient and coefficient $d$ through the interface even if coefficient $d$ is very small in term (c). On the other hand, the surface tension in the CSF model is estimated using the surface tension coefficient, for which the value is considerably larger than coefficient $d$. Thus, when considering a numerical simulation, the surface tension in the CSF model is larger than that of term (c).

The chemical potential $\mu[\mathrm{J} / \mathrm{mol}]$, which is important in investigating mass transfer, is defined by the functional derivative on the concentration $\eta\left[\mathrm{mol} / \mathrm{m}^{3}\right]$ with respect to the free energy $F$ as follows:

$\mu=\frac{\delta F}{\delta \eta}=\frac{\delta \psi}{\delta \eta} \frac{\delta F}{\delta \psi}$.

In the present study, the term $z_{i} e V_{e}$, which corresponds to the electrochemical potential, is derived from the variation $\delta F / \delta \eta$. Equation (44) is important not only in fluid motion but also in diffusion of mass.

\section{NUMERICAL SIMULATION}

\subsection{Governing Equation}

An interaction between two microbubbles was simulated while also accounting for the electrostatic potential. In the 
simulation, the validity of the multi-scale multiphase flow equation was qualitatively evaluated for the future realization of multi-scale simulations. If a chemical reaction and phase change are considered in two-phase flow analysis, the scale interactions of time and space among each phenomenon must be considered. However, such phenomena were not considered in this qualitative simulation for the sake of simplicity. Therefore, the simulation of the microbubble behavior focused on the local regions around the microbubble and was based only on the mesoscopic scale $\left(\nabla^{(1)}\right)$ using Eq. (42). For this assumption, we did not distinguish the velocity vectors and substituted the two terms on the left-hand side of Eq. (42) into $\mathrm{D} \boldsymbol{U} / \mathrm{D} t$. Finally, the following simplified equations were applied to the qualitative numerical simulation.

$$
\begin{aligned}
& \rho \frac{\mathrm{D} \boldsymbol{U}}{\mathrm{D} t}=-\boldsymbol{\nabla}^{(1)} \cdot \boldsymbol{T}+\varepsilon \boldsymbol{\nabla}^{(1)} \cdot\left(f_{e}(\psi) \boldsymbol{I}\right) \\
& -\varepsilon d \boldsymbol{\nabla}^{(1)} \psi\left(\boldsymbol{\nabla}^{(1)} \cdot \boldsymbol{\nabla}^{(1)} \psi\right)+\rho \boldsymbol{g} \\
& \boldsymbol{\nabla}^{(1)} \cdot \boldsymbol{U}=0 .
\end{aligned}
$$

For the second term on the right hand side of Eq. (45), only the electric term $f_{e}=c z_{i} e V_{e} \psi$ was considered; the first and second terms in Eq. (28) were omitted because phase change was not considered. Equations (45) and (46) were solved by the projection method [31]. The volume tracking method used for capturing the gas-liquid interface is based on the Multi-interface Advection and Reconstruction Solver (MARS) method [32]. This method is similar to the Piecewise Linear Interface Construction (PLIC) [33] algorithm and is based on the VOF method [34].

\subsection{Treatment of Order Parameter}

In the derivation of Eq. (42), the free energy was applied to the NS equation as the thermodynamic pressure, which corresponds to the surface tension. In Eqs. (27) and (28), parameters $a, b, c$, and $d$ are related to microscopic information such as intermolecular interaction. $\psi$ is an order parameter related to the concentration/density. For transportation of an interface with a finite thickness, using the profile function of the concentration or density as an a priori known function is the best way to characterize the system. However, in this study, transportation of the concentration/density profile function was not considered because this would make the numerical simulation complicated, especially when using an Eulerian approach. Our interfacial model was based on a lattice gas model, in which random molecular motion is modeled by a variable defined at a lattice point in a cubic lattice; the variable represents the existence of the molecules. Thus, the order parameter $\psi$ can be represented by the VOF in each bulk phase. The interfacial tracking method, MARS, tracks the interface by transporting the VOF. MARS has previously performed well using volume conservation as a macroscopic interfacial tracking method. Therefore, this method can also be a powerful tool for tracking the mesoscopic interface.

In the numerical simulation for this study, the mesoscopic interfacial tracking was performed by the order parameter $\psi$ in the third term on the right-hand side of Eq. (45). Concrete treatment of the parameter was modeled based on an analytical solution of the order parameter. The analytical solution of the order parameter was obtained by minimizing the free energy of Eq. (27) without the electric term. This analytical solution is known as a kink solution and is as follows.

$$
\psi_{0}(n)=\psi_{e} \tanh \left(\frac{n}{\delta_{0}}\right): \delta_{0}=\sqrt{\frac{2 d}{a}}, \psi_{e}=\sqrt{\frac{a}{b}}
$$

where $\delta_{0}$ is a correlation length corresponding to an interfacial thickness. This equation takes $\psi= \pm \psi_{e}$ at $n= \pm \infty$. In the present simulation, the order parameter was associated with the VOF based on the characteristics of Eq. (47).

$\psi=\psi_{e}\left(F_{A}-F_{B}\right)$ or $\psi_{e}\left(F_{B}-F_{A}\right)$

where $F_{i}(i=\mathrm{A}, \mathrm{B})$ is the VOF defined in a numerical cell. Here, $F_{A}$ and $F_{B}$ satisfy the relation $F_{A}+F_{B}=1 ; 0 \leq F_{A} \leq 1$ and $0 \leq F_{B} \leq 1$. In a strict sense, the order parameter minimizing Eq. (27) should be used in the present simulation by considering that energy is conserved. However, we assumed that Eq. (48) minimizes Eq. (27), and we used Eq. (48) in the simulation. The microscopic parameters $a$ and $d$ in Eqs. (27) and (28) include both the lattice width $l$ and the intermolecular potential. In this numerical simulation, the physical properties of the liquid were assumed to match water. Detailed discussion on the specificity of the water molecule is omitted here for the sake of brevity. The intermolecular potential was briefly evaluated using Eq. (43).

\subsection{Thermodynamic Pressure and Surface Tension Coefficient}

The third term on the right-hand side of Eq. (45) is expressed as follows:

$\boldsymbol{f}_{s}=-\varepsilon d \boldsymbol{\nabla}^{(1)} \psi\left(\boldsymbol{\nabla}^{(1)} \cdot \boldsymbol{\nabla}^{(1)} \psi\right)$.

The force $\boldsymbol{f}_{\mathrm{s}}$ surface tension. In this simulation, $\boldsymbol{\nabla}^{(1)} \cdot \boldsymbol{\nabla}^{(1)} \psi$, which was derived theoretically, was linearized for application in the numerical simulation as follows:

$$
\boldsymbol{f}_{s} \approx-\varepsilon d \nabla^{(1)} \psi \overline{\nabla^{(1)} \psi \mid}\left(\nabla^{(1)} \cdot \boldsymbol{n}\right)
$$

where the vector $\boldsymbol{n}$ is a unit normal vector. A simple average method was considered with respect to $\overline{\nabla^{(1)} \psi}$ by using $\left|\nabla^{(1)} \psi\right|$ defined at a numerical grid point $(i, j)$ and grid points around it. For example, in 2D simulations, $\overline{\nabla^{(1)} \psi \mid}$ is calculated by averaging the magnitude of $\left|\nabla^{(1)} \psi\right|$ defined at $(i, j),(i$ $+1, j),(i-1, j),(i, j+1)$, and $(i, j-1)$. In 3D simulations, $\overline{\nabla^{(1)} \psi \mid}$ is calculated by averaging $\left|\nabla^{(1)} \psi\right|$ defined at $(i, j, k),(i$ $+1, j, k),(i-1, j, k),(i, j+1, k),(i, j-1, k),(i, j, k+1)$, and $(i, j, k-1)$.

The parameter $d$ is related to the macroscopic surface tension coefficient through the following equation: 
$\sigma=\int_{-\infty}^{+\infty} d\left(\frac{\partial \psi_{0}}{\partial n}\right)^{2} d n \approx \int_{-\delta_{0} / 2}^{+\delta_{0} / 2} d\left(\frac{\partial \psi_{0}}{\partial n}\right)^{2} d n$

The macroscopic surface tension coefficient is defined under the condition of a flat interface and is estimated by integrating the gradient of the free energy along the normal direction perpendicular to the interface [10]. The parameter $d$ includes both the lattice width $l$ and the intermolecular potential. Equation (51) is calculated by using Eq. (47) analytically.

\subsection{Electrostatic force}

In our interfacial model, the existence of a contamination at the interface is represented by the existence of molecule A or B. In other words, the electric potential due to a contamination at the interface in bulk phase A (or B) is present when $F_{\mathrm{A}}=1$ (or $F_{\mathrm{B}}=1$ ). The specific formula of $f_{\mathrm{e}}$ is expressed as follows:

$f_{e}=\left.c z_{i} e V_{e}\right|_{i} \psi_{e}$

Strictly speaking, the Maxwell equations must be considered in Eq. (52) for the relationship between the electric charge and electric potential to hold. However, the law of conservation of charge was not considered in this study for the sake of simplicity. Therefore, the electrostatic potential $\left.V_{e}\right|_{i}$ in the bulk phases $\mathrm{A}$ and $\mathrm{B}$ through the interface was determined independently. The electrostatic potential of $\left.V_{e}\right|_{i}$ was estimated by the following exponential function:

$V_{e}(\boldsymbol{x})=V_{e 0} \exp \left(-\frac{\left|\boldsymbol{x}-\boldsymbol{x}_{\text {surface }}\right|^{2}}{C_{e}^{2}}\right)$.

In this equation, $C_{\mathrm{e}}$ is an arbitrary parameter that determines the effective region of the electrostatic potential, such as the Debye length. Position $\boldsymbol{x}$ is an arbitrary position of the bulk phase around a bubble. $\boldsymbol{x}_{\text {surface }}$ is the surface position of the bubble. The surface position was determined by the labeling method [3]. Substitution of Eq. (52) into the second term on the right-hand side of equation (45) gives the electrostatic force $\boldsymbol{F}_{\mathrm{e}}$ as follows:

$$
\boldsymbol{F}_{e}=-\varepsilon c z_{i} e \psi_{e} \boldsymbol{\nabla}^{(1)}\left(\left.V_{e}\right|_{i}\right),
$$

where $z_{\mathrm{i}} e$ is the electric charge of the contamination absorbed at the interface. $z_{\mathrm{i}} e$ is defined at the surface position. When an interfacial interaction was considered between bubbles 1 and 2, the electrostatic force was calculated by using the charge $z_{\mathrm{i}} e$ and electrostatic potential $V_{\mathrm{e}}$. For example, the electrostatic force at the interface of bubble 1 was estimated by the charge $z_{\mathrm{i}} e$ defined at the surface position of bubble 1 and the electrostatic potential $V_{\mathrm{e}}$ from bubble 2 .

\subsection{Numerical Condition}

A microbubble interaction was simulated in two dimensions (2D). The physical values used in this simulation were for water and air. Fig. (4) shows the numerical domain (301 $\times 301$ in the $x-y$ directions). Pressure-free conditions were taken as the boundary conditions at $X-$ and $X+$. At $Y-$ and $Y+$, the velocities were constant: $\left(U_{x}^{Y-}, U_{y}^{Y-}\right)=(0$, $\left.+2.0 \times 10^{-3}\right)$ at $Y-$ and $\left(U_{x}^{Y+}, U_{y}^{Y+}\right)=\left(0,-2.0 \times 10^{-3}\right)$ at $Y+$. The bubble radius, numerical mesh size, and time interval were $r=1.5 \times 10^{-6}[\mathrm{~m}], \mathrm{d} x=\mathrm{d} y=1.0 \times 10^{-7}[\mathrm{~m}]$, and $\mathrm{d} t=$ $0.5 \times 10^{-5}[\mathrm{~s}]$, respectively. In this simulation, two conditions were considered. In condition 1 , two microbubbles interacted with each other and the electrostatic potential was not considered. In condition 2 , the electrostatic potential $\left(V_{\mathrm{e}}=\right.$ $\left.-1.0 \times 10^{-5}[\mathrm{~V}]\right)$ was considered.

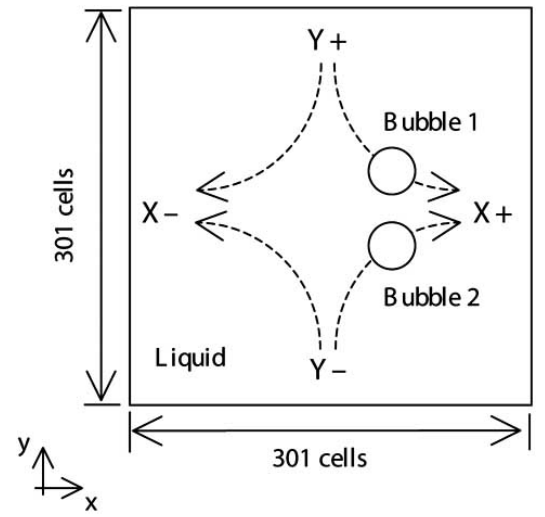

Fig. (4). Computational domain of microbubble interaction (2D).

In the present simulation, the microscopic parameters were set to $a=4.57 \times 10^{8}\left[\mathrm{~J} / \mathrm{m}^{3}\right], b=5.74 \times 10^{8}\left[\mathrm{~J} / \mathrm{m}^{3}\right], c=$ $1.33 \times 10^{28}\left[1 / \mathrm{m}^{3}\right]$, and $d=4.98 \times 10^{-11}[\mathrm{~J} / \mathrm{m}]$. The small parameter $\varepsilon$ was set to $\varepsilon=0.055$ [-]. Parameter $\varepsilon$ consists of the characteristic lengths $L$ and $\delta$ in Eq. (36). $L$ was set to 1 [ $\mu \mathrm{m}$ ], corresponding to the order of the Kolmogorov scale. $\delta$ was set to the averaged value of 10 and $100[\mathrm{~nm}]$; these values were from the van der Waals region and corresponded to the limiting thickness for film rupture $[35,36]$.

\section{RESULTS AND DISCUSSION}

Fig. 5 shows the results of $2 \mathrm{D}$ microbubble interaction. Figs. 5 (a) and (b) correspond to conditions 1 and 2, respectively. In Fig. 5 (a), the electrostatic potential of the bubble interface is not considered. Thus, the two microbubbles approach each other and coalesce immediately after coming in contact. Fig. 5 (b) shows the interaction of two microbubbles where the electrostatic potential is considered. The two microbubbles approach each other due to the hydrodynamic forces from the $Y+$ and $Y$ - directions and then interact with each other. However, the two microbubbles flow separately, maintaining a constant distance between them, and they do not coalesce.

Fig. (6) shows microbubble interaction at an interfacial electrostatic potential of $V_{\mathrm{e}}=-1.0 \times 10^{-4}[\mathrm{~V}]$. A liquid film is observed between the two microbubbles, as shown in Fig. 6 (a). Fig. 6 (b) shows the pressure distribution at the same time step. This figure shows that the pressure in the liquid film is lower than the bulk pressure around the microbubbles. This result indicates that the liquid in the bulk phase flows into the liquid film to conserve the continuity equation and maintain the thickness of the liquid film. The upper microbubble flows downwards and the lower microbubble 
(a)
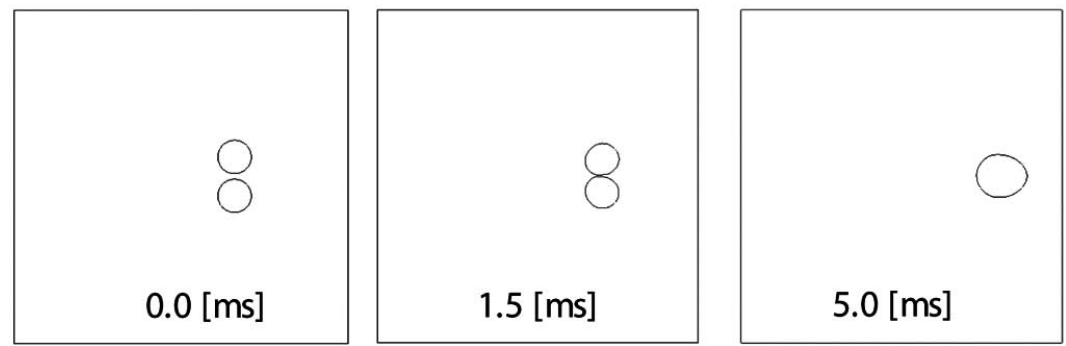

(b)
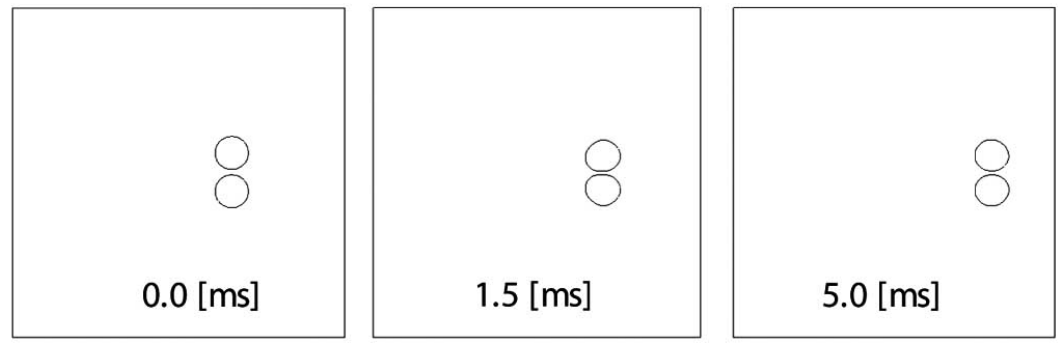

Fig. (5). Comparison of microbubble interaction. (a) Case 1: Electrostatic potential $V_{e}=0$ [V]. (b) Case 2: Electrostatic potential $V_{e}=-1.0 \times 10^{-5}[\mathrm{~V}]$

flows upwards due to the hydrodynamic force; i.e., the hydrodynamic force causes the two microbubbles to approach each other. In contrast, the electrostatic force at the interface causes the two microbubbles to separate. Eventually, a liquid film is formed between the two microbubbles due to force balance. The present numerical results may indicate that lubrication theory [37] based only on hydrodynamics cannot explain the presence of a liquid film between two microbubbles. That is to say, contaminations at the interface are important when interfacial interaction is considered.

(a)

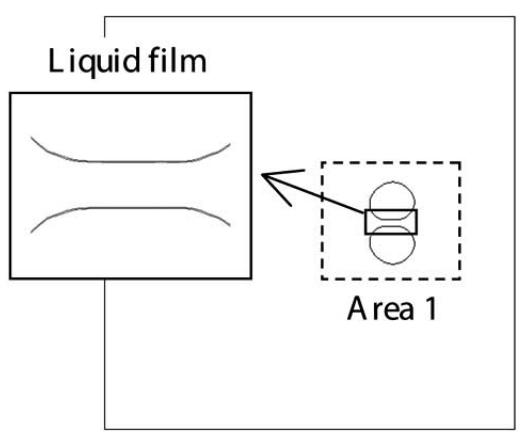

(b)

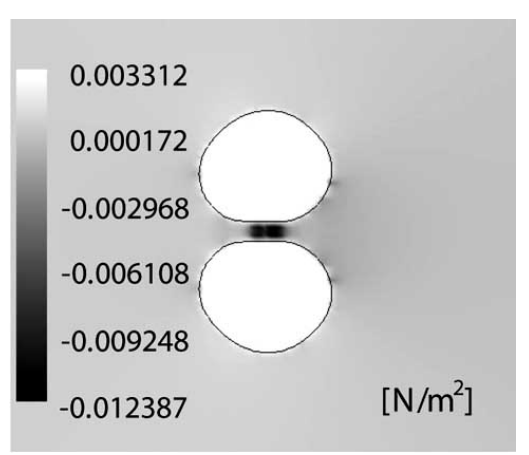

Fig. (6). Liquid film between two microbubbles (Electrostatic potential $\left.V_{e}=-1.0 \times 10^{-4}[\mathrm{~V}]\right)$. (a) Microbubble interaction. (b) Pressure distribution in Area 1 in (a).

\section{CONCLUSIONS}

In this study, a new mathematical model of the gas-liquid interface based on thermodynamics is developed using the assumption that the gas-liquid interface has a finite thickness similar to a thin fluid membrane. In particular, free energy is derived based on a lattice gas model that accounts for the electrostatic potential due to a contamination at the interface. Then, the free energy is incorporated into the NS equation using Chapman-Enskog expansion based on the multi-scale concept. The new governing equation proposed in this study has a theoretical multi-scale basis from the micro to the mesoscopic scales. In the numerical simulation, the $2 \mathrm{D} \mathrm{mi-}$ crobubble interaction was simulated to qualitatively evaluate the applicability of the multi-scale multiphase flow equation for the realization of a multi-scale simulation in the future. The following results were obtained:

[1] The microbubbles simply coalesced with each other when the electrostatic potential was not considered. In contrast, when the electrostatic potential was considered, a liquid film was formed between the two microbubbles, and the microbubbles flowed while maintaining a constant distance. The microbubbles did not coalesce.

[2] A liquid film was observed in our numerical simulation. The pressure in the liquid film was lower than the bulk pressure around the microbubbles. This implies that the liquid in the bulk phase flowed into the liquid film to conserve the continuity equation and maintain the thickness of the liquid film. This result may show that lubrication theory, based only on hydrodynamics, cannot explain the presence of a liquid film between two microbubbles. In other words, contaminations at the interface are important when interfacial interaction is considered.

Although the 2D numerical simulation performed in the present study serves only as a qualitative evaluation, we may be able to conclude that the multi-scale multiphase flow equation can be potentially used to simulate microbubble 
interaction. However, careful consideration of the time and space scales for the actual application in numerical simulations is essential when modeling both fluid motion and diffusion processes in the future.

\section{NOMENCLATURE}

\begin{tabular}{|c|c|c|}
\hline$E$ & $=$ & Electrostatic energy $[\mathrm{J}]$ \\
\hline$e$ & $=$ & Elementary charge $[\mathrm{C}]$ \\
\hline$F$ & $=$ & Free energy $[\mathrm{J}]$ \\
\hline $\mathrm{g}$ & $=$ & Acceleration due to gravity $\left[\mathrm{m} / \mathrm{s}^{2}\right]$ \\
\hline$H$ & $=$ & Hamiltonian $[\mathrm{J}]$ \\
\hline$h$ & $=$ & Plank's constant $[\mathrm{J} \cdot \mathrm{s}]$ \\
\hline$k_{B}$ & $=$ & Boltzmann constant $\left[\mathrm{m}^{2} \mathrm{~kg} / \mathrm{s}^{2} \mathrm{~K}\right]$ \\
\hline $\mathrm{L}$ & $=$ & Characteristic length of fluid motion [m] \\
\hline$l$ & $=$ & Lattice width in lattice gas model [m] \\
\hline$N$ & $=$ & Total number of lattice points [-] \\
\hline$P$ & $=$ & Pressure $\left[\mathrm{N} / \mathrm{m}^{2}\right]$ \\
\hline$T$ & $=$ & Temperature $[\mathrm{K}]$ \\
\hline$t$ & $=$ & Time $[s]$ \\
\hline$u$ & $=$ & Velocity vector $[\mathrm{m} / \mathrm{s}]$ \\
\hline$V_{\mathrm{e}}$ & $=$ & Electrostatic potential [V] \\
\hline$W_{i j}$ & $=$ & Intermolecular potential $[\mathrm{J}]$ \\
\hline$z_{\mathrm{i}}$ & $=$ & Valence [-] \\
\hline$\delta$ & $=$ & Characteristic length of the interface $[\mathrm{m}]$ \\
\hline$\eta$ & $=$ & Order parameter $\left[\mathrm{mol} / \mathrm{m}^{3}\right]$ \\
\hline$\mu$ & $=$ & Chemical potential $[\mathrm{J} / \mathrm{mol}]$ \\
\hline$\rho$ & $=$ & Density $\left[\mathrm{kg} / \mathrm{m}^{3}\right]$ \\
\hline$\sigma_{i}$ & $=$ & Existence of molecule A or B [-] \\
\hline$\sigma$ & $=$ & Surface tension coefficient $[\mathrm{N} / \mathrm{m}]$ \\
\hline$\tau$ & $=$ & Shear stress $\left[\mathrm{N} / \mathrm{m}^{2}\right]$ \\
\hline$\phi_{i}$ & $=$ & Local continuous function [-] \\
\hline$\phi$ & $=$ & Macroscopic continuum function [-] \\
\hline$\psi$ & $=$ & Order parameter [-] \\
\hline
\end{tabular}

\section{Subscripts}

$\begin{array}{lll}i, j & = & \text { Lattice points } \\ \text { int } & = & \text { Interface }\end{array}$

\section{REFERENCES}

[1] J. U. Brackbill, D. B. Kothe and C. Zemach, "Continuum method for modeling surface tension", J. Comput. Phys., vol. 100, pp. 335354, 1992.

[2] C. W. Hirt and B. D. Nichols, "Volume of fluid method for the dynamics of free boundaries", J. Comput. Phys., vol. 39, pp. 201225,1981

[3] Y. Matsumoto, T. Fukami, T. Kunugi and A. Serizawa, "Fluiddynamic structure of air-water bubbly flow with micro-bubbles", Proc. of the 4th International Conference on Multiphase Flow, 2001, CDROM-332.

[4] G. Tryggvason, B. Bunner, A. Esmaeeli, D. Juric, N. Al-Rawahi, W. Tauber, J. Han, S. Nas and Y. -J. Jan, " A front-tracking method for the computations of multiphase flow", J. Comput. Phys., vol. 169, pp. 708-759, 2001.

[5] A. Prosperetti and G. Triggvason, Computational methods for multiphase flow, Cambridge University Press, 2007.

[6] S. Osher and J. A. Sethian, "Fronts propagating with curvaturedependent speed: algorithms based on hamilton-jacobi formulations", J. Comput. Phys., vol. 79, pp. 12-49, 1988.

[7] T. Yabe, F. Xiao and T. Utsumi, "The constrained interpolation profile method for multiphase analysis", J. Comput. Phys., vol. 169, pp. 556-593, 2001.

[8] F. H. Harlow and J. E. Welch, "Numerical calculation of timedependent viscous incompressible flow of fluid with free surface", Phys. Fluids., vol. 8, pp. 2182-2189, 1965.

[9] J. W. Cahn and J. E. Hilliard, "Free energy of a nonuniform system, I. Interfacial energy", J. Chem. Phys., vol. 28(2), pp. 258-267, 1958.

[10] J. S. Rowlinson and B. Widom, Molecular theory of capillarity, Oxford University Press, 1984.

[11] B. Böttger, J. Eiken and I. Steinbach, " Phase field simulation of equiaxed solidification in technical alloys", Acta Mater, vol. 54, pp. 2697-2704, 2006.

[12] M. Plapp, "Three-dimensional phase-field simulations of directional solidification", J. Crystal Growth, vol. 303, pp. 49-57, 2007.

[13] R. S. Qin, E. R. Wallach and R. C. Thomson, "A phase-field model for the solidification of multicomponent and multiphase alloys", J. Crystal Growth, vol. 279, pp. 163-169, 2005.

[14] Y. Suwa and Y. Saito, "Phase field simulation of the effect of anisotropy in grain boundary energy on growth kinetics and morphology of grain structure", Mater. Trans., vol. 46(6), pp. 1-6, 2005.

[15] L. Onsager, "Reciprocal relations in irreversible processes. I.", Phys. Rev., vol. 37, pp. 405-426, 1931.

[16] L. Onsager, "Reciprocal relations in irreversible processes. II.", Phys. Rev., vol. 38, pp. 2265-2279, 1931.

[17] D. Jacqmin, "Calculation of two-phase Navier-Stokes flows using phase-field modeling”, J. Comput. Phys., vol. 155, pp. 96-127, 1999.

[18] D. Jacqmin, "Contact-line dynamics of a diffuse fluid interface", $J$. Fluid Mech., vol. 402, pp. 57-88, 2000.

[19] V. S. J. Craig, "Bubble coalescence and specific-ion effects", Curr Opin Colloid Interface Sci., vol. 9, pp. 178-184, 2004.

[20] C. L. Henry and V. S. J. Craig, "Ion-specific influence of electrolytes on bubble coalescence in nonaqueous solvents", Langmuir, vol. 24, pp. 7979-7985, 2008.

[21] S. Marčelja, "Short-range forces in surface and bubble interaction", Curr Opin Colloid Interface Sci., vol. 9, pp. 165-167, 2004.

[22] Jr. C. P. Ribeiro and D. Mewes, "The influence of electrolytes on gas hold-up and regime transition in bubble columns", Chem. Eng. Sci., vol. 62, pp.4501-4509, 2007.

[23] Y. H. Tsang, Y-H, Koh and D. L. Koch, "Bubble-size dependence of the critical electrolyte concentration for inhibition of coalescence", J. Colloid Interface Sci., vol. 275, pp. 290-297, 2004.

[24] R. R. Lessard and S. A. Zieminski, "Bubble coalescence and gas transfer in aqueous electrolytic solutions", Ind. Eng. Chem. Fundam., Vol. 10(2), pp. 260-269, 1971.

[25] S. Chapman and T. Cowling, The mathematical theory of nonuniform gases, $3^{\text {rd }}$ ed. Cambridge University Press, 1970.

[26] S. A. Safran, Statistical thermodynamics of surfaces, interfaces, and membranes, ADDISON-WESLEY PUBLISHING COMPANY, 1994.

[27] H. B. Callen, Thermodynamics and an introduction to thermostatistics, $2^{\text {nd }}$ ed. John wiley \& sons, 1985

[28] M. Fialkowski, A. Aksimentiev and R. Holyst, "Scaling of the euler characteristic, surface area, and curvatures in the phase separating or ordering systems", Phys. Rev. Lett., vol. 86(2), pp. 240-243, 2000.

[29] Y. Yonemoto, T. Kunugi and A. Serizawa, "Study on bubble shape interacted with vortex motion via mathematical approach", 58th Annual Meeting of the Division of Fluid Dynamics, vol. 50(9), p $250,2005$.

[30] J. N. Israelachvili, Intermolecular and surface forces, Academic Press, 1992.

[31] A. J. Chorin, Numerical solution of the Navier-Stokes equations, Math. Comput., vol. 22(104), pp. 745-762, 1968.

[32] T. Kunugi, MARS for Multiphase Calculation, Computational. Fluid Dynamics J., vol. 9, pp. 563-571, 2001. 
[33] D. L. Youngs, Numerical Methods for Fluid Dynamics, Academic Press, pp.273-468, 1982.

[34] C. W. Hirt, and B. D. Nichols, Volume of fluid method for the dynamics of free boundaries, J. Comput. Phys., vol. 39, pp. 201$225,1981$.

[35] D. G. Goodall, M. L. Gee, G. Stevens, J. Perera and D. Beaglehole, An investigation of the critical thickness of film rupture and drainage phenomena using dual wavelength ellipsometry, Colloids and Surfaces, vol. 143, pp. 41-51, 1998
[36] T. P. Witelski and A. J. Bernoff, Dynamics of three-dimensional thin film rupture,. Physica D, vol. 147, pp. 155-176, 2000

[37] O. Reynolds, On the Theory of Lubrication and its Application to Mr. Beauchamp Tower's Experiments, including an Experimental Determination of the Viscosity of Olive Oil, Philos. Trans. R. London, vol. 177, pp. 157-234, 1886.

Received: November 08, 2009

Revised: March 20, 2010

Accepted: March 22, 2010

(C) Yonemoto and Kunugi; Licensee Bentham Open.

This is an open access article licensed under the terms of the Creative Commons Attribution Non-Commercial License

(http://creativecommons.org/licenses/by-nc/3.0/) which permits unrestricted, non-commercial use, distribution and reproduction in any medium, provided the work is properly cited. 